\title{
Semi-Supervised Techniques for Mining Learning Outcomes and Prerequisites
}

\author{
Igor Labutov \\ Carnegie Mellon University \\ Machine Learning \\ Department \\ ilabutov@cs.cmu.edu
}

\author{
Yun Huang \\ University of Pittsburgh \\ Intelligent Systems \\ Program \\ yuh43@pitt.edu
}

\author{
Peter Brusilovsky \\ University of Pittsburgh \\ School of Information \\ Sciences \\ peterb@pitt.edu
}

\author{
Daqing He \\ University of Pittsburgh \\ School of Information \\ Sciences \\ dah44@pitt.edu
}

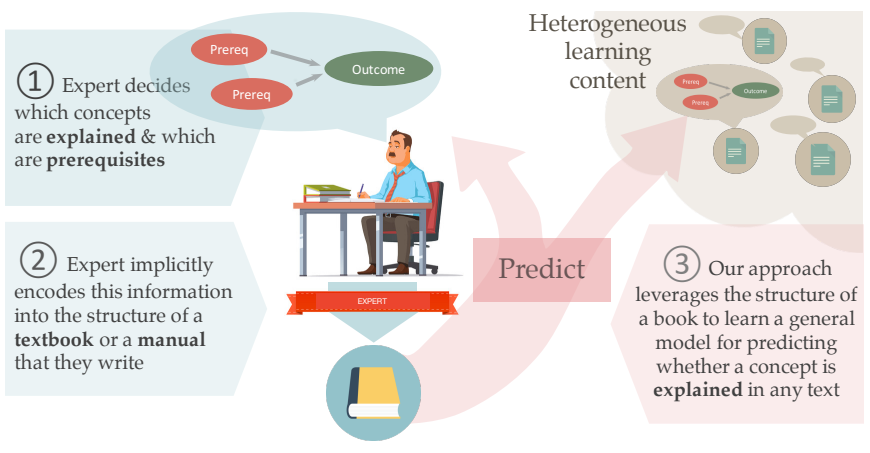

Figure 1: Textbooks are a natural source of supervision for learning a classifier to predict outcomes and prerequisites in educational texts. Our work explores the rich knowledge encoded in the structure of books to learn such classifiers.

which they assume as prior knowledge). In this paper, our focus is on exploiting textbooks as a rich source of supervision for training such models.

The problem with textbooks as a source of supervision, however, is that they are inherently noisy. Rarely do authors explicitly state which concepts they explain in each section (i.e., outcome concepts) and which they assume as prerequisites - the kind of annotation that could be directly exploited to learn a classifier for discriminating between outcome and prerequisite concepts. Even if they did, the annotation would likely be inconsistent across different textbooks and authors. Instead, authors tend to reveal this information in an implicit way, via indirect signals that manifest themselves in the structure of the textbook. Two such indirect signals that we exploit in this work are:

\section{- Supervision Source 1: Unit Sequence}

Our hypothesis is that the author usually explains a concept in one place (e.g., a chapter or a section).

- Supervision Source 2: Unit Titles

Our hypothesis is that the author of a textbook is more likely to include the concept's name in the title of a unit (e.g., chapter or section) if the concept is an outcome concept.

Neither of these signals, of course, will directly reveal whether a concept is an outcome or a prerequisite. A concept may sometimes be explained (i.e., an outcome) even if it's not mentioned in the title (Source 2). Similarly, knowing that a concept is explained in one place says nothing about where (Source 1). Nevertheless, our hope is that these signals provide a clue, even if a noisy or 
incomplete one, that can be exploited by a model that is intimately aware of how these indirect signals and the concept labels (i.e., outcome vs. prerequisite) are ultimately connected. For our task of learning a prerequisite/outcome classifier (that can be deployed on any document), this entails building a tractable statistical model that (i) can exploit these indirect signals as sources of distant supervision and (ii) can also take advantage of any potentially available labeled data.

Historically, adaptive educational hypermedia systems relied on the notion of outcomes and prerequisites in implementing adaptive navigation support techniques [1] that aid users in navigating educational content. Almost exclusively, however, the annotation of concepts as outcome or prerequisite has been performed by experts - a major obstacle to automating and scaling adaptive hypermedia systems to the web. We believe that by developing a technique for learning prerequisite/outcome classifiers from unlabeled textbooks, our work offers an important step forward in the field of Adaptive Tutoring. Because our method relies on natural sources of supervision (in contrast to expert annotation of outcomes and prerequisites), available in any textbook, our method can take advantage of the millions of available textbooks with little to no additional effort. The main contribution of our work is the framework for leveraging unlabeled textbooks as a source of supervision, more specifically:

- A model that takes advantage of the titles of textbook units as weak labels, in order to learn a prerequisite/outcome classifier.

- A model that uses the intuition that a concept is usually explained in one unit as a (soft) constraint during learning, in order to learn a prerequisite/outcome classifier.

- A comprehensive evaluation of the two models at the task of prerequisite/outcome classification on a corpus of six textbooks.

- An end-to-end evaluation of the two models at the task of predicting prerequisite documents within a web textbook annotated with a prerequisite graph.

\section{RELATED WORK}

Active research on concept analysis for electronic textbooks started with the emergence of adaptive textbooks in late 1990 [7]. Adaptive textbooks attempted to use adaptive navigation support techniques from the field of adaptive hypermedia [1] to guide the reader to the textbook sections that are most appropriate for his of her level of knowledge and learning goals. To model reader's knowledge, adaptive textbook used traditional overlay knowledge modeling approach $[4,15]$ developed in the field of Intelligent Tutoring Systems. With this approach, domain knowledge is modelled as set of knowledge components frequently called concepts and the current level of user knowledge is independently measured for each of these concepts. A learning goal could be also represented in terms of this domain model as a subset of concepts to master. In this context, a section in an electronic textbook could be recommended if we know, which concepts this section is explaining. Indeed, if some concepts explained in a section are a part of the current learning goal, but not yet mastered, the section become desirable and can be recommended to the user. To support this kind of personalization, adaptive textbooks allowed their creators to indicate the explained concepts (known as outcome concepts) for each section of the book.
However, outcome concepts alone are not sufficient for good personalization. While a page might present some desirable outcome concepts, it might not be ready for the reader to learn since some earlier concepts (usually referred as prerequisite concepts) have to be learned first. To address these problems, a number of early textbooks such as ISIS-Tutor [5], KBS-Hyperbook [13], and Multibook [19] used network domain models with prerequisite links directly connecting domain concepts. Unfortunately, this approach originally developed for knowledge sequencing in smalldomain Intelligent Tutoring Systems has not been scaling well for adaptive textbooks. Developing network domain models was notoriously hard. Besides, the nature of textbook explanations frequently required authors to refer to prerequisite concepts that were not anticipated by prerequisite links in the domain model.

To address this problem, some adaptive textbooks such as ELMART [6] and 2L670 [12] suggested to indicate prerequisite concepts directly for every textbook section. The model of an adaptive textbook where every section is indexed with a set of prerequisite and outcome concepts became very popular and was used by a number of platforms for authoring Web-based adaptive textbooks such as InterBook [2], AHA! [11], and NetCoach [20]. These platforms have been used to develop adaptive textbooks for a number of topics. The prerequisite/outcome model has been also used in some open corpus adaptive hypermedia (OCAH) systems [3]. The goal of an open corpus approach is to use in an adaptive way any online resource not originally considered by authors of an adaptive system. Indexing an online resource with a set of prerequisite and outcome concepts is one of the easiest ways to achieve this goal $[9,14]$.

The modern trend in OCAH system is gradual transition from manual content indexing to automatic concept extraction that allows a remarkable increase of scalability [18]. At the moment, many good concept-extraction approaches have been suggested. However, automatic separation of prerequisite and outcome concepts is still a problem with very few explored solutions. Past research explored two ideas to achieve this goal: the use of author's knowledge encapsulated in a sequential organization of a course [8] and more recently use of machine learning techniques to predict the class of each concept (prerequisite or outcome) on the basis of contextual and local features $[10,16,17]$. All of these methods, however, rely on datasets manually annotated with prerequisites and outcomes. Our key contribution in this work is a method for learning such classifiers from unlabeled textbooks, allowing us to potentially leverage millions of textbooks available in any discipline.

\section{METHOD}

In this section, we propose two probabilistic graphical models that we claim capture the intuition behind the two sources of distant supervision outlined in the previous section. A first step towards that, however, is to introduce the underlying classification model for predicting prerequisites/outcomes, which will be subjected to these two modes of distant supervision.

\subsection{Prerequisite/Outcome classifier}

The fundamental assumption underlying our work is that the authors of instructional content (e.g., a textbook) use different speech acts when referring to concepts that are outcomes vs. those that 
Semi-Supervised Techniques for Mining Learning Outcomes and Prerequisites

are prerequisites. Intuitively, we expect an explanation of a concept to involve acts such as defining or giving examples, which in turn are communicated via act-specific vocabulary (e.g., such as, is $a$, is an example of, etc.). More generally, we expect that the linguistic context surrounding the mentions of a particular concept is a good indicator to differentiate between whether the author is explaining that concept (i.e., outcome) or assuming it as prerequisite knowledge.

Naturally, this suggests a way of exploiting the linguistic context of a concept to automatically classify whether that concept is an outcome or a prerequisite. Using logistic regression as a model for classification, the probability that concept $i$ in unit $j$ (e.g., a chapter, section, etc.) is an outcome concept $\left(y_{i j}=\right.$ outcome $)$ can be expressed as follows:

$$
P\left(y_{i j}=\text { outcome } \mid \mathbf{x}_{i j}, \mathbf{w}\right)=\frac{1}{1+\exp \left(-\mathbf{w}^{T} \mathbf{x}_{i j}\right)}
$$

where $\mathbf{x}_{i j}$ represents the features of the context of concept $i$ in unit $j$ (e.g., chapter or section) and $y_{i j} \in\{$ outcome, prerequisite $\}$. The features encoded by $\mathbf{x}_{i j}$ may include things like neighboring words (unigrams, bigrams, etc.), grammatical roles (e.g., subject, object), and anything else that might be relevant in helping classify whether a concept is an outcome or a prerequisite. However, because $\mathbf{x}_{i j}$ summarizes the context of a concept across the entire unit, but a concept is usually mentioned multiple times and using multiple terms (e.g., EM and Expectation Maximization), it is important to be precise about how the contexts of the individual mentions of a concept are aggregated to build a single unit-level representation of that concept.

A simple and straightforward way to aggregate the context features across the mentions of that concept within a unit is a linear combination:

$$
\mathbf{x}_{i j}=\sum_{\substack{\text { mention } k \\ \text { of concept } i}} \mathbf{x}_{i j}^{(k)}
$$

where $\mathbf{x}_{i j}^{(k)}$ is a feature representation of the context of the $k^{t h}$ mention of concept $i$ in unit $j$. In all of our experiments in this work, we consider a single sentence to represent the context of a mention. Note that we may choose to transform the resulting linear combination to arrive at the final set of features for the concept (e.g., we may wish to binarize the features, as we do in our experiments).

\subsection{Learning $w$ via distant supervision}

In this work, our core task is to learn $\mathbf{w}$, the parameters of the logistic regression classifier. In principle, having learned $\mathbf{w}$, we hope to be able to deploy this classifier on educational content beyond the textbook on which it was trained - an important step towards understanding the educational content on the web.

To this end, we now turn to the problem of learning w. Recall that our core contribution are two methods that do not rely on the explicit annotation of prerequisites and outcome labels (i.e., $y_{i j}$ ) and instead employ weaker and more natural forms of supervision based on the universal structure of textbooks. Before describing the two models in technical detail, we briefly summarize how these models translate our high-level intuition about textbooks as a source of supervision, into the formal language of statistical modeling.

\section{Conference'17, July 2017, Washington, DC, USA}

\section{- SEQMODEL: Unit Sequence as a constraint}

Hypothesis: Our hypothesis is that the author typically explains a concept in one location (unit) within a textbook.

Model: This can be interpreted as an output-space constraint on the labels $y_{i j}$ across all units $j$ for a specific concept $i$. In the next section, we show that this constraint can be re-formulated as a tractable conditional mixture model

\section{- TitleModel: Unit Titles as weak labels}

Hypothesis: Our hypothesis is that the author of a textbook is more likely to include the concept's name in the title of a unit if that concept is an outcome concept, therefore making titles a source of weak supervision.

Model: We model titles as noisy labels, i.e., corrupted version of the true labels $y_{i j}$ which we treat as latent variables.

A note on outcomes and prerequisites. Note that by employing a binary classifier to distinguish between outcomes and prerequisites, we are making a strong assumption about a strict dichotomy, i.e., if a concept is not an outcome, it's necessarily a prerequisite and vice versa. In practice, of course, this is sometimes not the case. A concept may be referenced in passing, for example, without being either. At the same time, a concept in a single unit may appear as both, an outcome and a prerequisite. The assumption of a strict dichotomy is a convenient modeling assumption which often also holds in practice. In Section 5.5, we provide further justification to this assumption.

\subsection{Supervision source 1: Unit sequence}

We begin by recalling one of our core assumptions about textbooks: authors typically explain a concept in one location (unit) within a textbook. Translating this assumption to the language of our model in Equation 1, we can interpret it as a constraint over a sequence of prerequisite/outcome labels $y_{i j}$ across all units for every concept. To make this more explicit, consider the likelihood of observing all prerequisite/outcome labels $\mathbf{y}$ in a single textbook (i.e., for all concepts and units):

$$
P(\mathbf{y} \mid \mathbf{x}, \mathbf{w})=\prod_{\text {concept } i} \prod_{\text {unit } j} P\left(y_{i j} \mid \mathbf{x}_{i j}, \mathbf{w}\right)
$$

Estimating $\mathbf{w}$ is straight-forward when the prerequisite/outcome labels $\mathbf{y}$ are available - precisely not the case for a vast majority of textbooks. Therefore, instead, we are going to rely on a weaker form of supervision, in the form of a constraint on the output space (i.e., labels $\mathbf{y}$ ), encoding our intuition that a writer typically explains a concept in one unit. We can express the optimization problem for estimating $\mathbf{w}$, taking this assumption into consideration, as follows:

$$
\begin{aligned}
& \underset{\mathbf{w}}{\operatorname{maximize}} P(\mathbf{y} \mid \mathbf{x}, \mathbf{w}) \\
& \text { subject to } \sum_{j} y_{i j}=1, \forall i \\
& y_{i j} \in\{0,1\}, \forall i, j
\end{aligned}
$$

Directly introducing an arbitrary constraint into the learning process is not trivial. However, we observe that a constraint of this form can be reformulated as an unconstrained conditional mixture model by introducing a latent variable. Consider a mixture model 


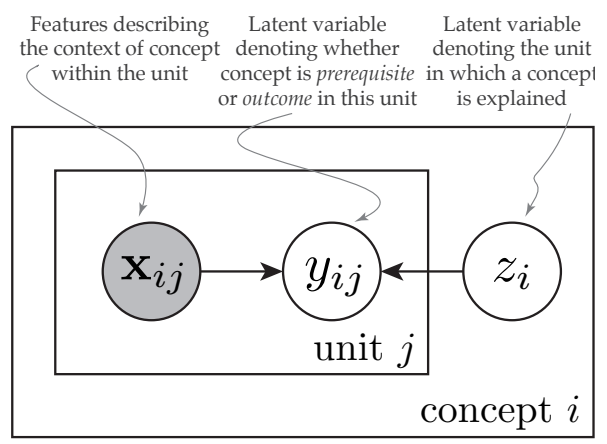

(a) SEQMOdEL: Unit Sequence

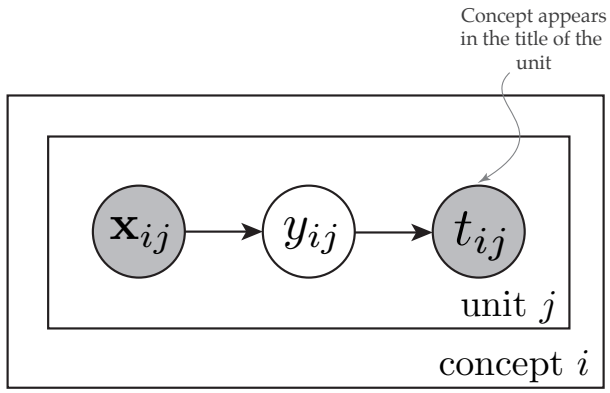

(b) TitheModel: Unit titles

Figure 2: Graphical model instantiations of the distant supervision signals naturally present in textbooks.

of $N$ components, where $N$ is the total number of units in the book. A component of this mixture can be expressed as follows:

$P\left(\mathbf{y}_{i} \mid z_{i}, \mathbf{x}_{i}, \mathbf{w}\right)=P\left(y_{i, z_{i}}=\right.$ outcome $\left.\mid \cdot\right) \prod_{j \neq z_{i}} P\left(y_{i j}=\neg\right.$ outcome $\left.\mid \cdot\right)$

where the latent variable $z_{i} \in\{1, \ldots, N\}$ indicates the unit where concept $i$ is an outcome concept. Note that the above expression only defines a probability of observing a specific sequence $\mathbf{y}_{i}$ in which the outcome unit is given by $z_{i}$. Defining a full likelihood over all possible sequences $\mathbf{y}_{i}$ (given $z_{i}$ ) would entail specifying a probability over all permutations of assignments to the individual $y_{i j}$ units in the sequence (i.e., for observations where the concept is explained in a unit other than the unit indicated by $z_{i}$ ). However, we avoid having to define the likelihood of these other observations by postulating that each concept is observed as an outcome precisely in the unit given by $z_{i}$. Thus, although the sequence $\mathbf{y}_{i}$ is not observed directly, we can nevertheless condition on it, in computing the posterior over $z_{i}$ (which will be used in the E-step described below).

The likelihood over all concepts and units in Equation 3 can then be expressed as follows, taking into account the latent variables:

$$
P(\mathbf{y} \mid \mathbf{x}, \mathbf{w})=\prod_{\text {concept }} \sum_{\text {unit } j} P\left(\mathbf{y}_{i} \mid z_{i}=j, \mathbf{x}, \mathbf{w}\right) P\left(z_{i}=j\right)
$$

which can be interpreted as a classic admixture model, on which we can bring to bear the standard EM inference procedure. A representation of this model as a probabilistic graphical model is given in Figure 2a. Note that by performing EM inference, the constraint in Equation 4 is implemented in a "soft" way, i.e., the result of inference is a distribution over units where a concept is explained. We briefly describe the key steps of the algorithm:

E-step: The E-step computes a posterior distribution over the latent variables $z_{i}$ (recall that $z_{i}$ is an indicator variable, indicating the unit in the book where a concept $i$ is explained). The expectation of the joint log-likelihood is then taken with respect to this posterior. After some algebra, we can show that the posterior over $z_{i}$ is:

$$
P\left(z_{i}=j \mid \mathbf{y}_{i}, \mathbf{x}_{i}, \mathbf{w}\right)=\frac{P\left(y_{i j}=\text { outcome } \mid \mathbf{x}_{i j}, \mathbf{w}\right)}{\sum_{j^{\prime}} P\left(y_{i j^{\prime}}=\text { outcome } \mid \mathbf{x}_{i j^{\prime}}, \mathbf{w}\right)}
$$

The posterior has a natural interpretation: the likelihood that a concept $i$ is explained (i.e., outcome) in unit $j$ is simply the normalized likelihood that the concept is an outcome concept in unit $j$ given by the logistic regression model in Equation 1.

M-step: The M-step is a straight-forward weighted logistic regression problem, where the weights correspond to the posterior over $z_{i}$. We refrain from additional technical detail due to space constraints.

\subsection{Supervision source 2: Unit titles}

We now shift our attention to the second core assumption about textbooks: authors are more likely to include the concept in a title if that concept is explained (i.e., outcome). Translating this assumption to the language of our model in Equation 1, we can interpret titles as "weak labels" that are noisy, but correlated with the true labels $y_{i j}$. Let $t_{i j} \in\{0,1\}$ be a binary variable that indicates whether or not the concept $i$ in unit $j$ appears in the title (e.g., Introduction to Expectation Maximization). The convenience of using $t_{i j}$ is that they are always observed, while the true labels $y_{i j}$, indicating whether the concept is a prerequisite or an outcome, are not (i.e., latent). Figure $2 \mathrm{~b}$ formalizes this intuition in a probabilistic graphical model.

Now, in addition to the parameters $\mathbf{w}$ of the logistic regression model, we need to introduce two additional parameters that connect the latent $y_{i j}$ to the observed $t_{i j}$. These parameters are $P\left(t_{i j} \mid\right.$ outcome $)$ and $P\left(t_{i j} \mid\right.$ prereq $)$, defined in Table 1 below.

\begin{tabular}{|c|c|}
\hline$P\left(t_{i j}=1 \mid\right.$ outcome $)$ & $\begin{array}{l}\text { Probability that the concept's name } \\
\text { appears in the title if that concept is an } \\
\text { outcome }\end{array}$ \\
\hline$P\left(t_{i j}=1 \mid\right.$ prereq $)$ & $\begin{array}{c}\text { Probability that the concept's name } \\
\text { appears in the title if that concept is a } \\
\text { prerequisite }\end{array}$ \\
\hline
\end{tabular}
Our intuition suggests that the author is more likely to include

Table 1: The two parameters connecting the appearance of a concept in the title and its prerequisite/outcome label.

the concept in the title if they intend to explain or introduce the concept, rather than assumes it as a prerequisite. However, we expect that the model discovers by itself, the degree to which the appearance of a title correlates with whether the concept is an outcome or a prerequisite (via the two parameters $P\left(t_{i j}=1 \mid\right.$ outcome) and $P\left(t_{i j}=1 \mid\right.$ prereq $\left.)\right)$. By decoupling the titles and the prerequisite/outcome labels within our model, we hope that the titles "nudge" the learning towards the correct model, while preventing them from contaminating it with noise. 
Semi-Supervised Techniques for Mining

Learning Outcomes and Prerequisites

We abstain from describing the E-step, as it is fairly straightforward to derive for this model. For learning the logistic regression parameters $\mathbf{w}$, the $\mathrm{M}$-step also reduces to the weighted logistic regression problem, similar to the model in the previous section. For the $P\left(t_{i j}=1 \mid\right.$ outcome $)$ and $P\left(t_{i j}=1 \mid\right.$ prerequisite $)$ parameters, interestingly, the M-step can be performed in closed form, while also providing some insight into the model. An updated estimate of $P\left(t_{i j}=1 \mid\right.$ outcome $)$ is computed as follows:

$$
\frac{\sum_{i, j} \delta\left[t_{i j}=1\right] P\left(y_{i j}=\text { out } \mid \cdot\right)}{\sum_{i, j} \delta\left[t_{i j}=1\right] P\left(y_{i j}=\text { out } \mid \cdot\right)+\sum_{i, j} \delta\left[t_{i j}=0\right] P\left(y_{i j}=\text { out } \mid \cdot\right)}
$$

where $\delta\left[t_{i j}=1\right]$ evaluates to 1 if concept $i$ appears in the title of unit $j$, and 0 otherwise (we omit the $\mathbf{x}_{i j}$ and $\mathbf{w}$ on the conditioning side of $P\left(y_{i j}=\right.$ out $\left.\mid \mathbf{w}, \mathbf{x}_{i j}\right)$ with to make the notation more compact). During the M-step, the above expression updates the estimate of $P\left(t_{i j}=1 \mid\right.$ outcome $)$ by computing the fraction of times that the concept's name appeared in the title while the model also believed that the concept was an outcome (weighted by the confidence of the model). The same argument applies to estimating $P\left(t_{i j}=1 \mid\right.$ prereq $)$.

During inference, we may choose to incorporate our prior belief about what $P\left(t_{i j}=1 \mid\right.$ outcome $)$ and $P\left(t_{i j}=1 \mid\right.$ prereq $)$ should be, which may help bias learning towards the right solution in the regime of little to no training data (less than $10 \%$ of data). For example, we may wish to encode our belief that $P\left(t_{i j}=1 \mid\right.$ prereq $)$ should be very small (i.e., probability that the concept appears in the title when it is a prerequisite). Our experiments experiments show that even with weak priors, proposed model can work reasonably well. A natural choice for incorporating this prior knowledge is via a Beta distribution, which is a distribution on probabilities. In Section 5, we will describe our choice for the Beta prior parameters and their impact on inference in more detail.

\section{EVALUATION}

A natural metric to evaluate a prerequisite/outcome classifier (and the model used to learn it) is a performance measure such as AUC on held-out data with labeled outcomes and prerequisites. To this end, we annotate six textbooks with prerequisite and outcome concepts in each unit, and use these textbooks for both training and evaluation. In Sections 5.1 and 5.2, we discuss an evaluation of the two proposed models (SEQModel and TitleModel) at the task of predicting outcome and prerequisite concepts.

Recall, however, that our ultimate goal is to train a prerequisite/outcome classifier that can be deployed to educational text outside of the textbooks that were used to train it. To this end, in Sections 5.4 and 5.5, we explore the ability of the learned models to generalize to other textbooks and tasks. In the following section, we briefly describe the datasets that we used in our evaluations.

\subsection{Datasets}

In total, we employ seven textbooks for our evaluation: Chris Bishop's Pattern Recognition and Machine Learning (from hereon referred to as PRML), five OpenStax ${ }^{2}$ textbooks in Biology, Anatomy,

\footnotetext{
${ }^{2} \mathrm{https}$ //openstax.org/
}

Conference'17, July 2017, Washington, DC, USA

Chemistry, Psychology and Economics, and an online statistics textbook from Rice University ${ }^{3}$, from hereon referred to as StatsBook. The PRML and OpenStax textbooks are used for evaluating the two models (SEQModel and TITLEMODEL) at the task of predicting outcomes and prerequisites. The StatsBook is used to evaluate the utility of the predicted prerequisite and outcome concepts at a downstream task of identifying prerequisite units (documents) in a prerequisite graph. We briefly describe each corpus, emphasizing the process by which we obtained the ground-truth annotation in each textbook.

4.1.1 Bishop's PRML. We employ the index of the textbook to identify concepts, and the units where they are explained. PRML conveniently provides explicit expert-annotation of the units (section or subsection) where concepts are explained, by highlighting the page corresponding to that unit in the index. To identify concept mentions in the text of the textbook, we perform manual terminology normalization, i.e., we compile a list of terms that correspond to the same concept (e.g., hidden variables, latent variables). The advantage of manual term normalization is that we are able to leverage substantially more contextual features around mentions that would otherwise be missed via exact term matching. While we perform normalization manually for this dataset, we expect that this task would be automated in the future.

4.1.2 OpenStax textbooks. OpenStax textbooks provide a similar type of expert-annotation as PRML. Each unit in an OpenStax textbook contains a list of keywords, corresponding to the concepts that are explained in that unit. We employ these keyword lists at the beginning of each unit as expert labels, labeling the concept corresponding to the keyword as an outcome concept. We do not, however, perform any manual terminology normalization, as we did in PRML. We will discuss the consequences of this in Section 5.3.

4.1.3 Rice Statistics book. The Rice University online Statistics textbook (StatsBook) is unique in that it comes with an expertcreated prerequisite graph. Every unit in the book is annotated with a set of other units that the author considers to be prerequisites (i.e., we can interpret this as a directed graph, where an edge exists between two units if one is a prerequisite of another). This type of annotation provides an opportunity to evaluate our model at a different, but related task, of identifying prerequisite units (documents). This task is closer to our broader goal of building on the results of the prerequisite and outcome classification, to create an automatic tutoring system that can guide learners through content.

Table 2 shows the statistics of these datasets.

\section{EXPERIMENTS}

In all experiments, we control the amount of ground-truth labels revealed to the model during training. This simulates the scenario where the annotator provides a small set of seed labels, and the model relies on both: (i) either the distributional constraint $\left(\mathrm{SE}_{\mathrm{E}}\right.$ QModel) or the weak labels (TitleModel) and (ii) the revealed ground-truth labels, in order to learn a prerequisite/outcome classifier. The focus of our evaluation, however, will be on the regime of very few labeled examples, which we consider to be more realistic in a practical setting. In all our evaluations, we perform 10 fold

\footnotetext{
${ }^{3}$ http://onlinestatbook.com/
} 


\begin{tabular}{lccccc}
\hline Textbook & Units & Terms & Instances & Out & $P\left(t_{i j} \mid\right.$ out $)$ \\
\hline Bishop PRML & 261 & 254 & 3,883 & 222 & $30 \%$ \\
\hline Biology & 47 & 637 & 3,188 & 637 & $1.9 \%$ \\
Anatomy & 28 & 1,063 & 4,850 & 1,063 & $1.1 \%$ \\
Chemistry & 22 & 234 & 1,358 & 234 & $3.0 \%$ \\
Psychology & 16 & 185 & 801 & 185 & $5.9 \%$ \\
Economics & 35 & 217 & 1,449 & 217 & $5.5 \%$ \\
\hline StatsBook & 113 & 340 & 1,879 & - & - \\
\hline
\end{tabular}

Table 2: Textbook dataset statistics

cross-validation, and report average AUC at the task of predicting outcome/prerequisite labels.

\subsection{Supervision source 1: Unit sequence}

We employ the following models in evaluating the SEQMODEL:

- Semi-supervised: SEqModel with EM-based learning, employing a variable amount of ground-truth labels during training.

- Supervised: A fully supervised logistic regression model, employing a variable amount ground-truth labels during training (and ignoring unlabeled data).

- Baseline: A naive baseline that considers the unit of the first occurrence of a concept in the book to be the unit where the concept is an outcome. This baseline stems from a strong intuition that an instructor would often explain the concept the first time they mention it. A logistic regression model is then trained on the dataset constructed using this assumption.

5.1.1 PRML evaluation. Figure 4a illustrates the results for the three models. We observe that the Semi-supervised model outperforms the Supervised model dramatically in cases with almost no ground-truth labels. We also observe that the effect of additional training data is fairly small, indicating that the simple distributional constraint on the labels based on the structure of the textbook is a powerful enough constraint to induce the labels without explicit annotation.

We illustrate two interesting posterior distributions over $z_{i}$, which represents the location in the textbook where term $i$ is explained. The illustrations are presented in Figure 3a and Figure $3 \mathrm{~b}$, and concern two concepts (conditional independence and latent variable, respectively). The $x$-axis in both figures corresponds to the linear ordering of the units within the textbook (left end-point corresponds to the beginning of the textbook), and blue stems reflect the probability that term $i$ is explained in a given unit (from the posterior distribution over $z_{i}$ ). Red stems correspond to the units where the term is annotated as an outcome (i.e., ground truth). In Figure 3a, observe that the term is not explained in its first appearance in the textbook, where the model correspondingly assigns low probability. Although conditional independence is given a cursory introduction in the first unit (where the posterior assigns a significant portion of the probability mass), the model correctly assigns the greatest probability mass to the chapter on graphical models, where the concept of conditional independence is explained thoroughly.

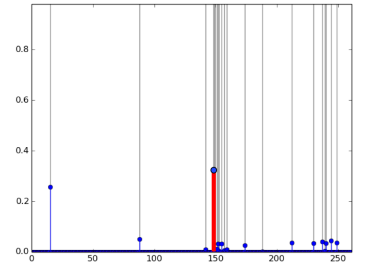

(a) Conditional independence

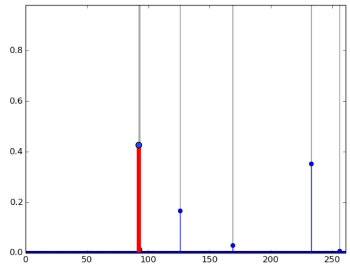

(b) Latent variable
Figure 3: Posterior distributions over $z_{i}$, i.e., location (unit) in the textbook where a concept is explained (i.e., outcome). The $x$-axis corresponds to a linear ordering of the units in the PRML textbook. Red color identifies the ground-truth unit where the concept is claimed to be explained by the author of the book. See Section 5.1.1 for a discussion.

5.1.2 OpenStax evaluation. We conduct the same evaluation on the five OpenStax textbooks, and present the results in Figure 5. We observe that the Semi-supervised model continues to excel over the Supervised model and the Baseline, specifically in the regime of few to no ground-truth labels.

\subsection{Supervision source 2: Unit titles}

In evaluating the TitLeModel, we also compare the Semi-supervised and fully Supervised models. Additionally, we implement a naive Baseline that uses titles as a source of direct supervision, i.e.:

- Baseline: A naive baseline that considers the presence of a concept in the title to be a deterministic indicator that the concept is an outcome concept. A logistic regression model is trained on this data, employing a variable amount of ground-truth labels during training.

We find that with the exception of very few labeled examples (e.g., 4 examples), the model is insensitive to a range of different priors during learning.

5.2.1 PRML evaluation. Figure $4 \mathrm{~b}$ illustrates the results for the three models. Similar to SEQModel, we observe that the Semisupervised approach substantially outperforms the Supervised approach in the regime of little to no ground-truth data. Although the Semi-supervised approach outperforms the Baseline with a smaller margin when having little to no ground-truth data, it significantly outperforms the Baseline consistently across all regimes.

5.2.2 OpenStax evaluation. We conduct the same evaluation of the TitreModel on the five OpenStax textbooks. We find that while the Semi-supervised approach performs comparably to the best-performing baseline, it does not, however, show a significant performance gain for all except one textbook (Economics). In the next section, we carry out a detailed analysis via a simulation study, in order to explain the difference in performance between the PRML and OpenStax datasets. 
Semi-Supervised Techniques for Mining Learning Outcomes and Prerequisites

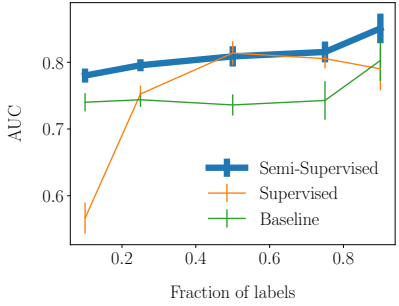

(a) SEQModel

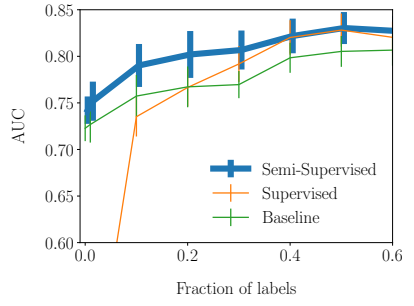

(b) TitleModel
Figure 4: Using the (a) unit sequence (SEQMODEL) and (b) unit titles (TITLEMODEL) of the textbook as sources of distant supervision in learning a prerequisite/outcome classifier. Evaluation was performed on Bishop's Pattern Recognition and Machine Learning textbook (PRML). The performance of our model (semi-supervised in blue) provides significant gains over a fully supervised model (orange) especially in low training data regime.

\subsection{Why is the performance of TITLEMODEL inconsistent between PRML and OpenStax?}

From our experiments on the PRML and OpenStax datasets, we observe that the TITLEMODEL has a noticeably different performance between the PRML and OpenStax datasets. From analyzing the statistics of the datasets in Table 2, our hypothesis is that the poorer performance of the TITLEMODEL stems from a very low proportion of titles in which the concept's name appears when that concept is explained (i.e., low P(title | outcome), last column in Table 2). For example, in PRML, approximately $30 \%$ of the units will mention the concept in the title if that concept is an outcome, in contrast to less than $6 \%$ in the OpenStax textbooks. To test our hypothesis, we conducted a simulation study, where we systematically generated synthetic "textbooks" with different $P$ (title | outcome) given a fixed $P$ (title | prereq), and compared the performance of the Semi-supervised model as a function of $P$ (title | outcome). Figure 6 summarizes the results.

We observe that the performance of the Semi-supervised model increases as a function of $P$ (title $\mid$ outcome), substantially improving over the Supervised model in the regime of $P$ (title | outcome $)>10 \%$, supporting our hypothesis that low $P($ title | outcome) in OpenStax may be the reason for the Semi-supervised model's low gains over the baselines. Two natural questions arise: (i) why does $P$ (title $\mid$ outcome) vary so substantially between the OpenStax and PRML textbooks and (ii) why does $P$ (title | outcome) affect the performance of the TitleModel? We provide our answers to these questions below.

Why does $P$ (title | outcome) vary so substantially between the OpenStax and PRML textbooks? Based on our analysis of the PRML and OpenStax data, we conclude that the lack of sufficient term normalization in the OpenStax data leads to low recall of concept mentions in the unit titles, and consequently an artificially lower $P$ (title | outcome) in the corpus. On the other hand, because $P R M L$ is processed manually (whereas terms are matched exactly in OpenStax), many more concept mentions are available
Conference'17, July 2017, Washington, DC, USA

in PRML, including mentions that appear in unit titles. To validate this claim, we have analyzed another textbook, Manning's Information Retrieval ${ }^{4}$, where we manually normalized terminology, and annotated concepts as prerequisites and outcomes ${ }^{5}$. We have found that $P$ (title | outcome) is between $20 \%$ and $40 \%$, depending on how the annotation of prerequisites and outcomes is performed. This further supports our hypothesis that term normalization is critical for obtaining a sufficient number of mentions of concepts in unit titles, and consequently a reasonable $P($ title $\mid$ outcome) required for the model to work well.

Why does $P($ title $\mid$ outcome) affect the performance of the TitleModel? When $P($ title $\mid$ outcome $)$ is low, there are simply too few titles to be exploited as weak labels during training ( $P$ (outcome) also tends to be low $(<25 \%)$ across all datasets). At the same time, because $P($ title $\mid$ prereq $)$ is also consistently low across textbooks, both $P$ (title | prerequisite) and $P$ (title | outcome) become difficult to distinguish, making titles a less discriminative signal in learning.

\subsection{How generalizable are the learned models?}

Recall that the core motivation for building a prerequisite/outcome classifier, is to be able to deploy it on educational content that exists outside textbooks, e.g., webpages. This raises an important question about the models proposed in this work: will a prerequisite/outcome classifier trained on one textbook, generalize to other content? To study this question, we carry out an evaluation where we train a prerequisite/outcome classifier on one textbook and evaluate it on the content of the remaining others. Although this task is still constrained to textbooks (because all of our annotated data consists of textbooks), the textbooks in our dataset are diverse, and present a reasonable challenge in testing the "generalizability" of the learned prerequisite/outcome models.

Figure 7 presents the results in a grid form, where rows and columns correspond to the textbooks that were used for training and testing respectively. We evaluate two versions of the SEQMODEL, trained with $10 \%$ and $90 \%$ of the ground-truth data, with AUC $\times 100$ displayed within the lower and upper parts of each cell respectively.

Based on these results, we draw the following two conclusions: (i) models learned using one source generalize to other content (even across diverse disciplines, such as Anatomy and Economics), and (ii) although models trained with more ground-truth data generalize better, they do so not by a large margin over models trained with only $10 \%$ of the data. Our key finding is that the learned models are general, and capture some universal linguistic features of what it means to explain a concept, regardless of the concept's domain.

\subsection{Can learned models predict prerequisite documents?}

Recall that one of our goals is to use the outcome and prerequisite labels as input to a system that would guide the user through educational content. To this end, an important question is how the prerequisite and outcome labels can be used to discover prerequisite documents.

\footnotetext{
${ }^{4} \mathrm{http}: / /$ nlp.stanford.edu/IR-book/

${ }^{5}$ the textbook is not yet in a sufficiently processed state to be used as one of the datasets
} 


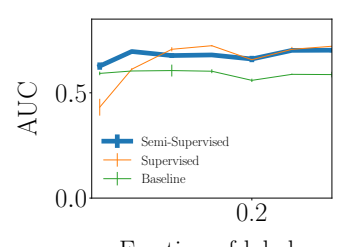

Fraction of labels

(a) Anatomy

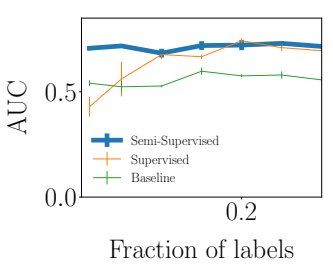

(b) Biology

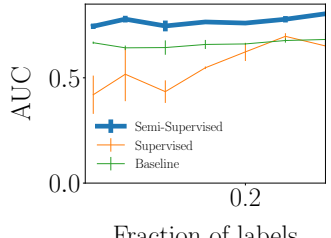

(c) Economics

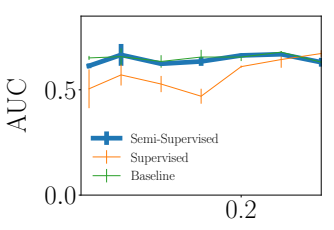

Fraction of labels

(d) Chemistry

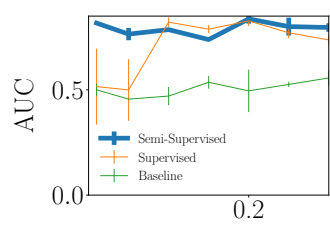

Fraction of labels

(e) Psychology

Figure 5: Using the sequential structure of the textbook as distant supervision in learning a prerequisite/outcome classifier. Evaluation was performed on 5 OpenStax textbooks. The performance of our model (semi-supervised in blue) provides significant gains over a fully supervised model (orange) especially in low training data regime.

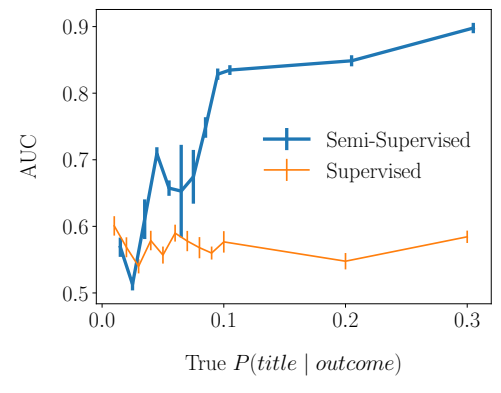

Figure 6: Simulation experiment varying the true $P($ title | outcome) used to generate the synthetic "textbooks". We show that a sufficiently large $P($ title $\mid$ outcome $)$ needs to be present in the dataset, for the model to be able to successfully exploit titles as weak labels during training.

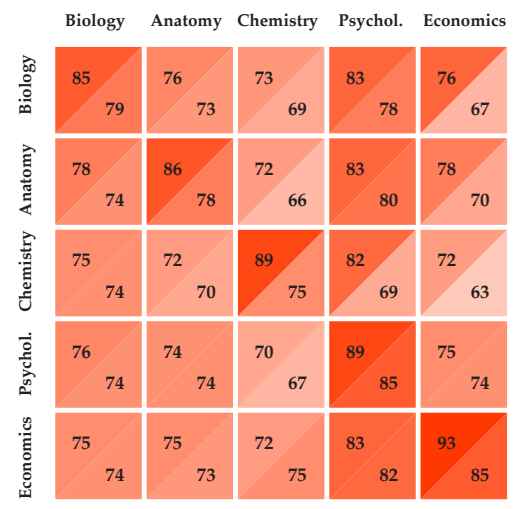

Figure 7: Evaluating the ability of the prerequisite/outcome classifiers trained on one textbook (row) to generalize to other textbooks (column). Upper and lower triangles correspond to the prerequisite/outcome classifier performance (AUC $\times 100)$ trained with the SEQMODEL using $90 \%$ and $10 \%$ of the data respectively.

We follow the work of [17], who have created a dataset annotated with a ground-truth prerequisite graph, i.e., specifying how units in a textbook (StatsBook) are connected based on their prerequisite/outcome relations. A key connection between the task of predicting prerequisite documents (units) and prerequisite concepts (our work), is defining a function that computes a "prerequisite score" as a function of a pair of units, based on their prerequisite and outcome concepts (e.g., returned by our prerequisite/outcome classifier). Due to space constraints, we refer the reader to [17], who define a number of such scoring functions. In this section, we use the scoring functions and baselines that they develop in order to evaluate the output of the prerequisite/outcome classifier, trained using an approach proposed in this paper.

The task of predicting a prerequisite document (unit) in a textbook (StatsBook) can be posed as a binary classification problem, i.e., discriminating between documents that are prerequisites vs. those that are not (as done in [17]). Furthermore, we can analyze this classification performance as a function of the prerequisite depth, i.e., the distance between the original document and its prerequisite in the prerequisite graph, e.g., Probability is a prerequisite of Expectation Maximization, but is typically separated by many units in a textbook (large prerequisite depth) vs. Maximum Likelihood and Expectation Maximization which may follow each other closely (low prerequisite depth).

Figure 8 shows the performance of predicting prerequisite documents as a function of prerequisite depth for three different scoring functions:

- Model: this scoring function incorporates the output of the prerequisite/outcome classifier.

- Baselines: two naive baselines that compute a scoring function without using the outputs of the prerequisite/outcome classifier. See [17] for more detail.

Note that we train the prerequisite/outcome classifier on a different textbook (PRML), and deploy the learned model to this task. Note that prerequisite depth ( $x$-axis in Figure 8$)$ should be interpreted cumulatively, i.e., as all units at some prerequisite depth that is lower than $x$.

Based on Figure 8, we make the following observations: incorporating the prerequisite/outcome classifications from the TITLEMODEL trained in a semi-supervised way improves performance (over the baselines) in predicting prerequisite documents. However, we find these performance gains exist only for a model trained with a small amount of ground-truth data ( $1 \%$ to $10 \%$ vs. $100 \%)$. We hypothesize that this is primarily due to the model trained with $100 \%$ of the ground-truth labels overfitting to the training textbook (PRML), and failing to generalize to StatsBook. Furthermore, in our experiments, prerequisite/outcome classifiers trained using the SEQMODEL did 


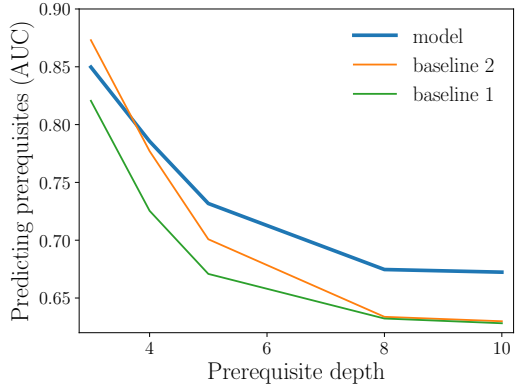

Figure 8: We evaluate the TITLEMODEL at the task of predicting prerequisite documents in the StatsBook corpus annotated with a prerequisite graph. The TITLEMODEL was trained on the PRML corpus with variable amount of ground-truth data. We find that fewer ground-truth labels (1\% to $10 \%$, with $10 \%$ shown in this figure) yields a model that is able to generalize better to this task. See Section 5.5 for details.

not substantially improve over the baselines in [17], which we too attribute to the model likely overfitting to the PRML dataset.

\subsection{Discussion}

A key take-away from our experiments and analysis is that the sequential structure of a textbook offers a more robust form of distant supervision than textbook titles. This stems from the fact that relying on the sequential nature of the book does not strongly depend on the quality and coverage of the extracted terms, as we have seen to be the case for a model that relies on titles as weak supervision. A model that relies on titles as weak supervision, will require a sufficiently high recall in term extraction, so that a sufficiently large proportion of title concepts are extracted. Any model that closely relies on terminology extraction will likely suffer from similar limitations.

More important towards our broader vision of deploying the learned prerequisite/outcome classifiers outside of the books on which they were trained, we demonstrate that the proposed methods yield classifiers that generalize to broader educational content.

\section{CONCLUSION}

Our key contribution in this paper is the idea and a practical demonstration of how textbooks can be leveraged as a rich source of supervision to train models for identifying learning outcomes and prerequisites in educational texts. We proposed two models that exploit two simple observations about how textbooks naturally encode experts' knowledge implicitly via the structure of the books they write, in order to learn the prerequisite/outcome classifiers without explicit annotation. This means that our method can utilize millions of published textbooks as training data.

Furthermore, we demonstrate that the prerequisite/outcome classifiers learned using our models generalize across diverse learning material (different disciplines), which implies that these models successfully capture some domain-agnostic essence of what it means to explain a concept. We believe that our work opens doors to a new generation of adaptive tutoring systems that make sense of the diverse educational content on the web (by understanding outcome and prerequisite concepts) and provide personalized guidance to learners to achieve their learning goals.

To encourage further research in this direction, we release all datasets annotated as part of this work, as well as all code that can be used to reproduce our results. They can be download at_this_url.

\section{REFERENCES}

[1] Peter Brusilovsky. 1996. Methods and techniques of adaptive hypermedia. User Modeling and User-Adapted Interaction 6, 2-3 (1996), 87-129.

[2] Peter Brusilovsky, John Eklund, and Elmar Schwarz. 1998. Web-based education for all: A tool for developing adaptive courseware. In Seventh International World Wide Web Conference. 291-300.

[3] Peter Brusilovsky and Nicola Henze. 2007. Open corpus adaptive educational hypermedia. In The Adaptive Web: Methods and Strategies of Web Personalization. Springer-Verlag, Berlin Heidelberg New York, 671-696.

[4] Peter Brusilovsky and Eva Millán. 2007. User models for adaptive hypermedia and adaptive educational systems. In The Adaptive Web: Methods and Strategies of Web Personalization. Springer-Verlag, Berlin Heidelberg New York, 3-53.

[5] Peter Brusilovsky and Leonid Pesin. 1998. Adaptive navigation support in educational hypermedia: An evaluation of the ISIS-Tutor. Fournal of Computing and Information Technology 6, 1 (1998), 27-38.

[6] Peter Brusilovsky, Elmar Schwarz, and Gerhard Weber. 1996. ELM-ART: An intelligent tutoring system on World Wide Web. In Third International Conference on Intelligent Tutoring Systems, ITS-96. Springer-Verlag, 261-269.

[7] Peter Brusilovsky, Elmar Schwarz, and Gerhard Weber. 1997. Electronic textbooks on WWW: from static hypertext to interactivity and adaptivity. Educational Technology Publications, Englewood Cliffs, New Jersey, 255-261.

[8] Peter Brusilovsky, Sergey Sosnovsky, Michael Yudelson, and Girish Chavan. 2005. Interactive Authoring Support for Adaptive Educational Systems. In 12th International Conference on Artificial Intelligence in Education, AIED'2005. 96-103.

[9] Cristina Carmona, David Bueno, Eduardo Guzmn, and Ricardo Conejo. 2002 SIGUE: Making Web Courses Adaptive. In Second International Conference on Adaptive Hypermedia and Adaptive Web-Based Systems (AH'2002). SpringerVerlag, 376-379.

[10] Sahar Changuel, Nicolas Labroche, and Bernadette Bouchon-Meunier. 2015. Resources Sequencing Using Automatic Prerequisite-Outcome Annotation. ACM Transactions on Intelligent Systems and Technology 6, 1 (2015), 6.

[11] Paul De Bra and Licia Calvi. 1998. AHA! An open Adaptive Hypermedia Architecture. The New Review of Hypermedia and Multimedia 4 (1998), 115-139.

[12] Paul M. E. De Bra. 1996. Teaching Hypertext and Hypermedia through the Web. fournal of Universal Computer Science 2, 12 (1996), 797-804.

[13] Nicola Henze, Kabil Naceur, Wolfgang Nejdl, and Martin Wolpers. 1999. Adaptive hyperbooks for constructivist teaching. Künstliche Intelligenz 4 (1999), 26-31.

[14] Nicola Henze and Wolfgang Nejdl. 2001. Adaptation in open corpus hypermedia. International fournal of Artificial Intelligence in Education 12, 4 (2001), 325-350.

[15] Yun Huang, Michael Yudelson, Shuguang Han, Daqing He, and Peter Brusilovsky. 2016. A Framework for Dynamic Knowledge Modeling in Textbook-Based Learning. In 24th Conference on User Modeling Adaptation and Personalization. ACM, 141-150.

[16] Sonal Jain and Jyoti Pareek. 2013. Automatic extraction of prerequisites and learning outcome from learning material. International fournal of Metadata, Semantics and Ontologies 8, 2 (2013), 145-154

[17] Igor Labutov and Hod Lipson. 2016. Web as a textbook: Curating Targeted Learning Paths through the Heterogeneous Learning Resources on the Web. In 9th Intl. Conf. Educational Data Mining (EDM 2016). 110-118.

[18] Seamus Lawless, Lucy Hederman, and Vincent Wade. 2008. Enhancing Access to Open Corpus Educational Content: Learning in the Wild. In The 19th ACM Conference on Hypertext Hypermedia. 167-174.

[19] A. Steinacker, C. Seeberg, K. Rechenberger, S. Fischer, and R. Steinmetz. 1999 Dynamically generated tables of contents as guided tours in adaptive hypermedia systems. In 11th World Conference on Educational Multimedia and Hypermedia. AACE, 640-645.

[20] Gerhard Weber, Hans-Christian Kuhl, and Stephan Weibelzahl. 2002. Developing adaptive internet based courses with the authoring system NetCoach. In $\mathrm{Hy}$ permedia: Openness, Structural Awareness, and aptivity. Springer-Verlag, Berlin, $226-238$. 